Voix et Images

voixetimages

\title{
Découvrir, fonder, survivre : les romans historiques de Laure Conan
}

\section{Katherine A. Roberts}

Volume 24, numéro 2 (71), hiver 1999

Poésie québécoise et histoire littéraire

URI : https://id.erudit.org/iderudit/201433ar

DOI : https://doi.org/10.7202/201433ar

Aller au sommaire du numéro

Éditeur(s)

Université du Québec à Montréal

ISSN

0318-9201 (imprimé)

1705-933X (numérique)

Découvrir la revue

Citer cet article

Roberts, K. A. (1999). Découvrir, fonder, survivre : les romans historiques de Laure Conan. Voix et Images, 24(2), 351-371. https://doi.org/10.7202/201433ar
Résumé de l'article

Cet article propose une analyse à la fois féministe et narratologique des trois romans historiques de Laure Conan : À l'oeuvre et à l'épreuve (1891), L'oublié (1905) et la sève immortelle (1925). De ces trois textes se dégage une perspective féminine sur l'épopée de la colonisation du Canada français. En effet, entre le premier et le troisième roman, le récit « masculinisant » de la Conquête s'efface au fur et à mesure que l'essence féminine sublimée (la Vierge) est remplacée par des femmes en chair et en os. En reconfigurant les notions de courage et d'héroïsme, Laure Conan nuance l'histoire convenue de la Nouvelle-France. 


\title{
Découvrir, fonder, survivre: les romans historiques de Laure Conan*
}

\author{
Katherine A. Roberts, Queen's University
}

\begin{abstract}
Cet article propose une analyse à la fois féministe et narratologique des trois romans bistoriques de Laure Conan: À l'œuvre et à l'épreuve (1891), L'oublié (1905) et La sève immortelle (1925). De ces trois textes se dégage une perspective féminine sur l'épopée de la colonisation du Canada français. En effet, entre le premier et le troisième roman, le récit "masculinisant" de la Conquête s'efface au fur et à mesure que l'essence féminine sublimée (la Vierge) est remplacée par des femmes en chair et en os. En reconfigurant les notions de courage et d'béroüsme, Laure Conan nuance l'bistoire convenue de la Nouvelle-France.
\end{abstract}

Le roman québécois naît au passé. Mécontents du présent qui n'offre aucune prise à la sublimation romanesque, les romanciers du $\mathrm{XIX}^{\mathrm{e}}$ siècle se tournent vers l'histoire de la Nouvelle-France, vers la Conquête ou la Rébellion de 1837 ; bref, vers les moments charnières de la survivance de leur collectivité. Munis des travaux de François-Xavier Garneau et de l'abbé Ferland, influencés par la revalorisation romantique du passé et du "génie national" (Volkgeist), ces premiers écrivains optent pour le genre historique, développé et popularisé par Walter Scott ${ }^{1}$.

Or, le choix d'un sujet historique de la part du très petit nombre de femmes québécoises qui écrivent à la fin du siècle s'avère plus complexe.

* Cette recherche a été rendue possible grâce à une bourse de doctorat du Conseil de recherches en sciences humaines et sociales du Canada, organisme que je tiens à remercier de son soutien.

1. Pour une étude récente de l'importance des romans de Walter Scott dans la littérature du $\mathrm{XIX}^{\mathrm{e}}$ siècle, on lira avec profit Ina Ferris, The Achievement of Literary Autbority, Gender, History and the Waverly Novels, Ithaca, Cornell University Press, 1991. Rappelons que l'influence scottienne s'est étendue jusqu'au Québec. Voir David Hayne, "The Historical Novel and French Canada", thèse de doctorat, Université d'Ottawa, 1945, p. 68-70; Eva-Marie Kröller, "Walter Scott in America, English Canada and Québec: A Comparison ", Canadian Review of Comparative Literature, vol. VII, $\mathrm{n}^{\circ} 1$, 1980 , p. 32-46; Maurice Lemire, Les grands thèmes nationalistes du roman bistorique canadien-français, Québec, Presses de l'Université Laval, 1970, p. 239-245. 
Dans un article sur les rédactrices des premiers périodiques féminins Joséphine Marchand (Le coin $d u$ feu) et Robertine Barry (Le journal de Françoise) - Mary Jean Green décrit la stratégie qu'adoptent plusieurs collaboratrices de l'époque pour faire face à un climat décidément hostile aux femmes qui écrivent. Vue par les élites clérico-politiques comme une force subversive qui contredit l'idéologie dominante, cette écriture féminine tâche d'assurer sa survie en exagérant les vertus dites féminines du sacrifice et de la modestie ${ }^{2}$. Les premières journalistes du Canada français ont également su tirer profit du climat de nationalisme culturel effervescent, où la composition littéraire était un devoir patriotique, pour encourager des sujets d'inspiration historique. Ce faisant, elles encourageaient la lecture chez les filles et créaient un public, et donc un marché, pour une littérature féminine encore embryonnaire. C'est justement dans le contexte de cette double contrainte culturelle - l'écriture des femmes se voyant obligée, pour exister, de réinscrire la femme dans son rôle de gardienne de la langue française et de la foi catholique - qu'il faut situer Laure Conan.

Première écrivaine de cette littérature en émergence, reconnue surtout pour avoir inauguré, avec Angéline de Montbrun (1881), le genre psychologique, Laure Conan s'adonne également au roman historique, publiant $\hat{A}$ l'œuvre et à l'épreuve (1891), L'oublié (1905) et La sève immortelle (1925). Quoique bien reçus par la critique journalistique de l'époque, ces trois récits n'ont suscité que des analyses sommaires ${ }^{3}$. Plutôt que de réhabiliter des textes souvent qualifiés de "pâles romans" ", cet article cherchera les traces d'une perspective féminine dans un genre qui, malgré les efforts de ces premières journalistes, demeure essentiellement masculin au Québec ${ }^{5}$. Que se passe-t-il quand une femme décrit la quête d'origines historico-mythiques, symbolisée par la mise en scène de la

2. Mary Jean Green, "The "Literary Feminists" and the Fight for Women's Writing in Québec", Revue d'études canadiennes/Journal of Canadian Studies, vol. XXI, $\mathrm{n}^{\circ} 1$, 1986, p. 134.

3. Voir Jules S. Lesage, Notes biographiques. Propos littéraires, Montréal, Édouard Garand, 1931, p. 171-180; Maurice Lemire, op. cit., p. 40-44 et p. 150-155; Dennis Duffy, Sounding the Iceberg: An Essay on Canadian Historical Novels, Toronto, ECW, 1986, p. 25-27. La seule exception à cette règle est l'article de Fernand Roy, "Laure Conan et l'institution littéraire: d'Angéline de Montbrun à La sève immortelle: rupture malheureuse ou étonnante continuité?", Claudine Potvin et Janice Williamson (dir.), Women's Writing and the Literary Institution/L'écriture au féminin et l'institution littéraire, Edmonton, University of Alberta, Research Institute for Comparative Literature, 1992, p. 189-198. En étudiant la conception de l'écriture mise en ouvre dans Angéline de Montbrun, l'auteur consacre un paragraphe de son analyse à chacun des trois romans historiques.

4. Gilles Marcotte, Une littérature qui se fait, Montréal, Bibliothèque Québécoise, 1994, p. 41 .

5. A part Laure Conan, seulement deux autres écrivaines de cette même période se sont essayées au genre historique: Adèle Bibaud (Avant la conquêle. Épisode de la guerre de 
Nouvelle-France? Dans quelle mesure Laure Conan souscrit-elle à la version établie de l'épopée de la colonisation du Canada français?

\section{Au-delà de «l'influence » de l'abbé Casgrain : la parole autoritaire}

De tous les lieux communs qui entourent l'œuvre et la personne de Laure Conan - "une femme timide, discrète, tourmentée, qui cachait son vrai nom (Félicité Angers) sous un pseudonyme ${ }^{6}$-, celui qui explique son passage du roman psychologique au roman historique est peut-être le plus pernicieux. Selon la légende, Laure Conan aurait sollicité une préface auprès de l'abbé Henri-Raymond Casgrain pour la première édition d'Angéline de Montbrun. Pour Fernand Roy et Louise Milot, il s'agit bien évidemment d'une stratégie:

[U]n éloge du leader national lui assurait la notoriété et constituait en même temps un imprimatur. Ce n'étaient pas là précautions inutiles, et encore moins s'agissant d'une personne qui vivait hors des grands centres intellectuels, d'une femme ni mariée ni religieuse, et qui pratiquait un genre encore mal vu en 1880 au Canada français 7 .

Il est vrai que dans cette préface astucieuse et élogieuse, celui qui se nomme le père de la littérature québécoise souligne, quoique brièvement, la "physionomie trop européenne" d'Angéline de Montbrun:

Sa pensée habite plus les bords de la Seine que ceux du Saint-Laurent. On regrette de ne pas rencontrer assez de pages vraiment canadiennes, telles que celle du pèlerinage d'Angéline au tombeau de Garneau. Notre littérature ne peut être sérieusement originale qu'en s'identifiant avec notre pays et ses habitants, qu'en peignant nos mours, notre histoire, notre physionomie: c'est sa condition d'existence ${ }^{8}$.

Profitant de l'occasion pour diffuser sa rhétorique et promouvoir la bonne littérature canadienne ${ }^{9}$, Casgrain offre un conseil qu'il aurait donné à tout écrivain sérieux de l'époque, ce qui n'est pas en soi suffisant pour légitimer la réaction combien paternaliste de la critique, illustrée ici dans l'introduction d'À l'ouvre et à l'épreuve de Roger Le Moine:

Pour satisfaire aux exigences de l'abbé Casgrain et des critiques du temps, Laure Conan s'oriente vers le roman historique, après Angéline de Montbrun [...] elle continue de mettre l'accent sur les personnages - comme si elle

1757 [1904] et Les fiancés de St-Eustache [1910]), et Gaëtane de Montreuil (Fleur des ondes [1912]). Voir les listes qu'ont établies Hayne, op. cit., p. 180-184 et Lemire, op. cit., p. 254-260.

6. Gilles Marcotte, op. cit., p. 38.

7. Fernand Roy et Louise Milot, "La réception critique du premier roman québécois au féminin ", Angéline de Montbrun, Montréal, Bibliothèque Québécoise, 1990 [1881], p. 10.

8. Henri-Raymond Casgrain, Euvres complètes, tome I, Montréal, Beauchemin, 1875, p. 413.

9. Voir à ce sujet Réjean Robidoux, Fonder une littérature nationale, Ottawa, Éditions David, 1994, p. 181-198. 
écrivait un autre roman psychologique - et non sur les événements euxmêmes ${ }^{10}$.

En somme, ces trois romans historiques sont perçus comme des travestissements forcés au nom de la littérature nationale, comme des textes qui ne contiennent pas la voix authentique, présente dans son premier roman.

Une étude du sentiment national dans l'œuvre de Laure Conan, visible en filigrane dans Angéline de Montbrun et rendu plus explicite dans sa pièce Si les Canadiennes le voulaient! (1886), nécessite que soient pris en compte ces trois textes d'inspiration historique, qui constituent par la structure de leurs intrigues et par leurs configurations symboliques une réflexion de taille sur le passé du Canada français et sur le rôle considérable qu'y ont joué plusieurs figures féminines héroïques. En fait, des études récentes ont souligné non pas la rupture subite entre Angéline de Montbrun et la production romanesque ultérieure, attribuée à l'influence de l'abbé Casgrain, mais une sorte de filiation qui relie toute son œuvre. Pour Louise Milot et Fernand Roy, la clé se trouve dans la place qu'occupe, dans le cheminement d'Angéline, la figure de François-Xavier Garneau, premier historien canadien-français. Dans la troisième partie d'Angéline de Montbrun intitulée "Feuilles détachées", Angéline évoque les moments où elle lisait des passages de l'Histoire du Canada de Garneau en compagnie de son père et souligne également l'admiration qu'avait $M$. de Montbrun pour "celui qui a tant fait pour l'honneur de notre nom ${ }^{11}$ ". Elle entreprend même un pèlerinage à sa tombe au cimetière Belmont: "[...] devant le monument de [leur] historien, le généreux sang de [s]es ancêtres coula chaud dans [s]es veines." ( $A M, 167)$ Dans l'imaginaire d'Angéline, Garneau semble cumuler deux rôles: celui de père substitut, tel que le suggèrent Roy et Milot, et celui de modèle de fierté nationale. Il symbolise le sacrifice et la dévotion à la patrie. Pour Laure Conan, Garneau fait figure à la fois d'autorité en matière d'histoire ${ }^{12}$ et d'instigateur de. son écriture. En tirant de l'oubli les héros du passé, il inspire la mise en fiction de l'histoire, à laquelle elle se livrera par la suite.

D'autres passages d'Angéline de Montbrun montrent également l'orientation de ses écrits futurs. Par exemple, on a fait peu de cas d'une

10. Roger Le Moine, "Introduction d'À l'œuvre et à l'épreuve", Euvres romanesques, tome II, Montréal, Fides, coll. "Nénuphar", 1975, p. 9.

11. Laure Conan, Angéline de Montbrun, Euvres romanesques, tome I, Montréal, Fides, coll. "Nénuphar ", 1974, p. 167. Désormais, les références à cet ouvrage seront indiquées par le sigle $A M$, suivi du folio, et placées entre parenthèses dans le texte.

12. Selon Roger Le Moine, Laure Conan a certainement puisé dans l'Histoire du Canada pour écrire À l'œuvre et à l'épreuve et L'oublié. Dans ce dernier roman, un long passage de l'ouvrage de Garneau est même cité en note afin de décrire les guerres autochtones. Voir Laure Conan, L'oublié, CEuvres romanesques, tome II, op. cil., p. 269. Désormais, les références à cet ouvrage seront indiquées par le sigle $O$, suivi du folio, et placées entre parenthèses dans le texte. 
lettre de P. $\mathrm{S}^{* * *}$, insérée dans la dernière partie du roman. Le missionnaire $\mathrm{y}$ rend hommage à $\mathrm{M}$. de Montbrun et décrit les joies et les misères de sa vie parmi les Chippeways ( $A M, 176-180)$. Ce poignant et instructif témoignage n'est qu'un avant-goût du récit que nous réserve Charles Garnier dans À l'œuvre et à l'épreuve. Dans un autre ordre d'idées, on sait qu'Angéline, comme le dit son amie Mina Darville dans ses lettres, a la nostalgie du bon vieux temps: elle "aime les vaillants et les grands coups d'épée" $(A M, 24)$. Cousine du duc de Lévis, vaincu par James Murray à Montréal en 1760, "elle aime le souvenir de ces jours où la voix de Lévis retentissait sonore, et elle s'indigne contre les Anglais qui n'ont pas rougi de lui refuser les honneurs de la guerre" $(A M, 34)$. Sous la plume d'Angéline elle-même, cette fascination presque naïve pour le temps des hommes forts s'élargit pour englober l'héroïsme et la fidélité de son aieule, $\mathrm{M}^{\mathrm{me}}$ de Montbrun, qui a perdu son mari et deux fils dans la bataille contre les Anglais à Sainte-Foy:

Ce printemps de $1760, \mathrm{M}^{\text {me }}$ de Montbrun laboura elle-même sa terre, pour pouvoir donner du pain à ses petits orphelins. Vaillante femme!

J'aime me la représenter soupant fièrement d'un morceau de pain noir, sa rude journée finie. J'ai d'elle une lettre écrite après la cession, et trouvée parmi de vieux papiers de famille, sur lesquels mon père avait réussi à mettre la main lors de son voyage en France. C'est une fière lettre.

"Ils ont donné tout le sang de leurs veines, dit-elle, en parlant de son mari et de ses fils, moi, j'ai donné celui de mon cœur; j'ai versé toutes mes larmes. Mais ce qui est triste, c'est de savoir le pays perdu ${ }^{13}$." $(A M, 166)$

Pour Mary Jean Green, la vie des femmes comme $M^{\text {me }}$ de Montbrun fournit à Angéline une ascendance spirituelle dans la mesure où celle-ci possède, comme elles, des qualités de fidélité et de sacrifice ${ }^{14}$. De plus, les propos d'Angéline - j'aime me la représenter - témoignent d'une volonté de conjuguer fiction et histoire en redonnant vie aux héroïnes moins connues de la Nouvelle-France. On verra plus loin l'influence primordiale de cette ascendance sur les actions des protagonistes féminins dans les deux derniers romans historiques. L'existence de "modèles" en sol canadien permet aux femmes des générations futures de réclamer pour elles-mêmes une partie de l'aventure du Nouveau Monde.

Le passage du roman psychologique au roman historique chez Laure Conan s'explique par divers facteurs. S'agit-il uniquement d'une. stratégie de légitimation comme dans le cas des rédactrices des premiers périodiques féminins, mais qui aurait eu ici un effet pervers, à savoir celui de discréditer sa fiction historique? On peut déjà répondre qu'Angéline de Montbrun contient les éléments d'une poétique de l'histoire qui deviendra

13. C'est moi qui souligne.

14. Mary Jean Green, "Laure Conan and Madame de La Fayette: Rewriting the Female Plot ", Essays on Canadian Writing, no 34, 1987, p. 56. 
la qualité maîtresse de toute son ouvre. Or, la décision de Laure Conan de mettre sa plume au service du roman historique, quoique liée indubitablement à cette stratégie de légitimation et à un vif intérêt pour l'histoire du Canada français, devrait aussi être vue comme une quête d'autorité discursive. C'est ce que je tenterai de montrer brièvement.

La narratologue américaine Susan Sniader Lanser soutient que, málgré l'ambivalence d'une écrivaine face aux institutions de pouvoir, l'acte d'écrire un roman et de le publier constitue une quête d'autorité discursive, une volonté d'être entendue, d'exercer une influence. Dans son excellente analyse de la corrélation entre l'essor du roman et les différents types de voix narratives qui sont "disponibles" aux femmes à des moments divers de l'histoire, Lanser fait une distinction entre la voix narrative privée et la voix publique: l'une et l'autre correspondent à deux types de conscience narrative dans la construction de l'autorité textuelle. Dans le cas de la voix privée, l'instance narrative s'adresse à un narrataire qui est aussi personnage dans l'histoire (comme dans Angéline de Montbrun), tandis que, dans celui de la voix publique, l'instance narrative s'adresse à un narrataire en dehors du texte, analogue au lecteur réel. La situation narrative qui fait autorité est hétérodiégétique, publique et autoréflexive. Le narrateur s'y engage dans des "moments extrareprésentationnels" sous la forme de réflexions, de commentaires ou de jugements sur le monde extérieur à la diégèse. De tels commentaires élargissent la sphère d'autorité fictive pour inclure le référent "non fictionnel", ce qui permet à l'écrivain de participer, de l'intérieur du monde fictif, aux débats de l'heure, tant culturels que littéraires et intellectuels. La "voix personnelle" se limite aux narrateurs qui racontent leurs propres histoires; elle ne comporte pas les mêmes privilèges que la voix auctoriale (authorial voice). Ces différences rendent la voix personnelle moins redoutable pour l'auteur-femme que la voix d'une narratrice auctoriale, puisque celle-ci revendique les pouvoirs que lui confère un savoir sur le monde, tandis que la narratrice personnelle ne peut réclamer que la validation de son seul témoignage ${ }^{15}$.

La théorie de Lanser permet d'éviter la sous-estimation du choix d'une narration publique à l'époque à laquelle écrivait Laure Conan. Malgré le fait que ses romans historiques semblent offrir moins de matière à une analyse féministe - leurs héroïnes doublement dévouées à la Vierge et à la patrie perdent leur aspect révolutionnaire comparées à Angéline de Montbrun qui refuse et le mariage et le couvent À l'œuvre et à l'épreuve, L'oublié et La sève immortelle fournissent à l'écrivaine l'occasion d'exploiter la voix autoritaire propre au genre

15. Susan Sniader Lanser, Fictions of Authority: Women Writers and Narrative Voice, Ithaca/London, Cornell University Press, 1992, p. 17 et passim. 
historique, ce qui lui permet de "participer " plus ouvertement au discours des hommes en recréant l'histoire nationale.

\section{Le discours messianique}

Analyser le discours national et comprendre la portée symbolique de l'œuvre de Laure Conan nécessitent un examen des courants idéologiques qui traversent le paysage intellectuel de l'époque. Les trente dernières années du $\mathrm{XIX}^{\mathrm{e}}$ siècle, où culmine le projet de créer une littérature nationale, sont imprégnées d'une idéologie qu'on désigne du nom de "messianisme canadien-français". Ce nationalisme singulier s'inscrit dans une conception de l'ordre social où l'absolu religieux et la différence linguistique garantissent la survie du peuple francophone en tant qu'héritier d'une glorieuse aventure, celle de la Nouvelle-France ${ }^{16}$. Dans Le Québec et ses historiens de 1840-1920. La Nouvelle-France de Garneau à Groulx, Serge Gagnon étudie les rapports entre la structure sociale canadienne-française du $\mathrm{xIx}^{\mathrm{e}}$ siècle et la structure du récit des historiens concernant les premiers temps de la colonie. Relevant la forte présence de figures cléricales dans le domaine de l'historiographie, il identifie cette période du Régime français en Amérique du Nord comme la clé de voûte de l'idéologie messianique. Plusieurs historiens opposent au sombre tableau des premiers jours de la domination anglaise de brillantes descriptions de la Nouvelle-France ${ }^{17}$. Selon Jean Blain, que Gagnon cite à l'appui, "la quasi absolue dépendance des historiens canadien-français à un type de sources [...] tend beaucoup plus à éclairer un idéal de colonisation qu'une réalité coloniale [...] nos historiens ont été [...] amenés à faire l'histoire de ce que devrait être la Nouvelle-France ${ }^{18}{ }_{n}$. Une telle lecture historique a fait en sorte qu'encore au début $\mathrm{du} \mathrm{xx}^{\mathrm{e}}$ siècle "la reconstitution du passé canadien-français est moins la recherche empirique d'un donné [...] que le dégagement et le rappel d'une continuité de sens, d'un retour à des archétypes qui puissent fonder un destin ${ }^{19}$ ".

Toute représentation d'une époque révolue devient chez l'écrivain une relecture, une réinterprétation guidée par les courants idéologiques en circulation au moment de sa rédaction et, surtout, par la nature des documents historiques qui lui sont accessibles. La fiction emprunte ses vérités historiques à des documents qui ont leurs propres réorganisations

16. Réjean Beaudoin, Naissance d'une littérature. Essai sur le messianisme et les débuts de la littérature canadienne-française (1850-1890), Montréal, Boréal, 1989, p. 8-9.

17. Serge Gagnon, Le Québec et ses bistoriens de 1840-1920. La'Nouvelle-France de Garneau à Groulx, Québec, Presses de l'Université Laval, 1978, p. 15.

18. Ibid., p. 3 .

19. Fernand Dumont, "Du début du siècle à la crise de 1929: un espace idéologique", Idéologies au Canada Français 1900-1929, Québec, Presses de l'Université Laval, 1974, p. 9 . 
symboliques, leur propre importance sémantique. Ce qu'il importe de retenir ici, c'est la présence des nombreuses couches d'interprétation historique au service du nationalisme messianique auxquelles est exposée Laure Conan au moment où elle écrit ses romans. Sa poétique historique ne saurait être comprise en dehors de ce climat envahissant d'histoire providentielle. Il ne s'agit pas ici de réduire les romans historiques à une mise en fiction de l'idéologie dominante, mais plutôt d'étudier la façon dont elle a réussi à modifier le projet "virilisant" de la fondation de la Nouvelle-France véhiculé par cette idéologie en mettant l'accent sur les exploits des femmes à la fois religieuses et laïques, réelles et fictives.

\section{Ensemencer, effacer}

Au début d'à l'ouvre et à l'épreuve, nous sommes en 1625, en France; la jeune orpheline Gisèle Méliand quitte Port-Royal-des-Champs, où elle a passé une grande partie de son enfance, pour regagner la demeure de ses parents adoptifs. Elle y attendra le retour du fils cadet, Charles Garnier, dont elle est amoureuse, les deux enfants ayant été destinés l'un à l'autre depuis longtemps. Charles revient d'un voyage en Italie épris d' espérances plus hautes ${ }^{20}$ "et décide de suivre l'exemple de ses deux frères aînés en entrant en religion. Ce choix exige de Gisèle l'ultime sacrifice. Pour la gloire de Dieu, elle accepte la tâche qui consiste à obtenir du père de Charles le consentement dont le jeune homme a besoin : elle convainc ses parents de le laisser devenir missionnaire. Voilà pourquoi la deuxième moitié du roman se déroule au Canada et raconte les dernières années du père Charles Garnier, martyrisé par les Iroquois en 1650 .

Cette dernière partie du texte témoigne d'un nouveau type de vision historique où, en l'absence de descriptions des lieux, le lecteur est initié à l'état d'esprit des missionnaires qui ont voulu évangéliser la NouvelleFrance. L'absence totale de renseignements historiques sur la première partie de la vie de Charles Garnier permet à la romancière de reconstituer sa vie de famille et d'introduire le personnage de la fiancée. Quant à la vie du père en Nouvelle-France, Conan reste fidèle aux sources; elle reproduit même une lettre authentique adressée par Garnier au père LeJeune et cite L'instruction pour les pères de notre compagnie qui seront envoyés aux Hurons de Brébeuf ${ }^{21}$, ce qui confère au récit un cachet d'authenticité et d'autorité.

Faisant revivre l'époque hérö̈que des missionnaires, ce premier roman glorifie sans réserve la Nouvelle-France. En ce sens, le personnage

20. Laure Conan, À l'œuvre et à l'épreuve, CEuvres romanesques, tome II, op. cit., p. 74. Désormais, les références à cet ouvrage seront indiquées par le sigle $O E$, suivi du folio, et placées entre parenthèses dans le texte.

21. Voir Roger Le Moine, "Introduction" d'À l'œuvre et à l'épreuve, op. cit., p. 15. 
de Champlain, ami du père de Charles Garnier, est une figure charnière. De retour en France pour organiser les affaires de la colonie, Champlain, accompagné par sa femme, habite quelques jours chez les Garnier. Le discours que l'auteure prête au fondateur de la Nouvelle-France pour raconter ses exploits héroïques souligne à quel point l'aventure canadienne est une aventure masculine, voire "masculinisante":

Oui, j'implanterai dans le sol canadien le vieil honneur et la vieille foi... si profondément... que, pour en arracher jamais ces germes d'héroïsme, il faudra tout bouleverser, tout détruire ${ }^{22}$. $(O E, 43)$

Champlain chassera la barbarie tandis que la France, représentée par l'allégorie du guerrier chrétien, s'avançant dans ces immenses régions d'outre-mer, "jett[era] la semence de vie" $(O E, 54)$. Son fondateur, "couché au pied d'un arbre, [s]on arquebuse à portée de [l] main" (OE, 54), bâtira sa ville de Québec et en fixera la destinée. Celui qui a "bu aux fleuves inconnus, qui a marché sur les feuilles entassées par les siècles" $(O E, 45)$ éblouit ses interlocuteurs avec des descriptions hyperboliques du paradis terrestre, d'une colonie entièrement composée d'hommes, honorée par la puissante bénédiction de la Sainte Vierge. Mais si la figure de la Vierge Marie plane sur la colonie - elle sera omniprésente dans L'oublié - , la terre vierge de la Nouvelle-France n'est pas compatible avec la femme réelle. Y ayant déjà emmené une fois sa femme, Champlain décide de façon irrévocable qu'elle ne l'accompagnera plus jamais: "Il y a trop de privations à supporter... trop de dangers à courir. " $(O E, 57)$

L'entretien entre Champlain et le père Brébeuf, qui est venu le voir chez les Garnier, renforce l'idée de l'entreprise de la colonie comme une affaire de surhommes. Le corps du religieux est ainsi décrit:

Bon connaisseur de la force physique, Champlain ne pouvait s'empêcher d'admirer aussi la carrure du missionnaire: Taillé en athlète! pensait-il, en regardant ses épaules puissantes, son cou large, musculeux [...]. Il complimenta gaiement le jésuite, l'assurant que sa force lui serait bien utile et lui vaudrait l'admiration des sauvages. (OE, 63-64)

La rencontre entre ces deux personnages historiques sert aussi à mettre sur un pied d'égalité l'œuvre du fondateur et celle du missionnaire. La précieuse sève française requise pour coloniser se transformera, dans l'économie symbolique du texte, en sang des martyrs. Brébeuf se réjouit déjà de la possibilité de souffrir la plus horrible des morts: "Il faut du sang pour que la semence de vie germe et prenne racine." $(O E, 65)$ Sous la plume de Laure Conan, Champlain prend les allures d'une figure christique pour qui le but de la colonisation sera uniquement religieux. "Le salut d'une âme vaut mieux que la conquête d'un empire" $(O E, 66)$, dit-il à Brébeuf. Celui-ci répète ainsi d'autres paroles du fondateur: "Les

22. C'est moi qui souligne. 
rois ne doivent songer à s'emparer des pays idolâtres que pour les soumettre à Jésus-Christ." (OE, 66) Il va sans dire qu'une telle vision, qu'on pourrait qualifier de prosélytisme religieux, obscurcit les visées économiques et mercantiles de la fondation de la Nouvelle-France. Mais en faisant de Champlain un missionnaire catholique, Laure Conan ne fait que s'inscrire dans le courant de l'historiographie, voire du discours dominant de la société canadienne-française du XIX ${ }^{\mathrm{e}}$ où un culte en l'honneur de Champlain est largement répandu ${ }^{23}$.

Le personnage de Champlain remplit en effet une fonction plus poétique qu'historique chez Laure Conan. En tant que héros chrétien, il inspire Charles Garnier qui se sacrifie pour la gloire de Dieu. Dans la dernière partie du roman, l'accent mis sur la vie des vaillants missionnaires tels que le père Garnier entraîne cependant l'abandon du discours patriotique et religieux, véhiculé en grande partie par Champlain. En fait, on devine ici la présence de toute une série de tensions idéologiques qui laissent émerger une vision plus sombre des réalisations en terre canadienne que celle à laquelle souscrit son fondateur. Dans le portrait du missionnaire, héros d'un courage inouï, on peut suivre les traces d'un certain amour-propre: "Charles ne songe plus qu'à sa perfection" $(O E, 61)$, dit son père à Champlain. Son refus du monde terrestre afflige durement tant Gisèle que ses parents: le père meurt de chagrin. Dans sa première lettre à Gisèle après son arrivée au Canada, Garnier exprime sa joie d'avoir été choisi par Dieu:

C'est dans cette chère chapelle que j'ai célébré ma première messe en la Nouvelle-France: une messe d'apôtre, car c'était la fête de saint Barnabé. Et, Gisèle, avec quelle triomphante allégresse, j'ai lu ces paroles de mon maitre: "Je vous ai choisis et tirés du monde." $(O E, 131)$

Le piège de l'amour-propre trouve son écho dans l'avertissement de Brébeuf:

Il est vrai, disait-il, que l'amour de Dieu a la force de faire ce que fait la mort, c'est-à-dire de nous détacher des créatures et de nous-mêmes; néanmoins ces désirs que nous ressentons de coopérer au salut des infidèles ne sont pas toujours des marques assurées de cet amour épuré; il peut y avoir quelquefois un peu d'amour-propre et de recherche de nous-mêmes, si nous regardons seulement le bien et le contentement qu'il y a de mettre des âmes dans le ciel, sans considérer mûrement les peines, les travaux et les difficultés qui sont inséparables de ces fonctions évangéliques. ( $O E, 134-135)$

Sans ce passage de Brébeuf, les indices d'amour-propre dans les lettres de Garnier n'auraient pas de signification particulière. Mais mises en rapport avec les conseils du premier apôtre des Hurons, elles tendent, selon moi, à remettre en question la pureté d'âme du jeune religieux et ternissent ainsi l'histoire glorieuse des missionnaires, ouvrant une brèche dans l'épopée de la colonisation du Canada français.

23. Serge Gagnon, op. cit., p.151. 
Les interventions narratives (ou moments extrareprésentationnels) de ce premier roman, quoique rarissimes, sont des plus intéressantes pour mon propos dans la mesure où elles ne s'accordent pas tout à fait avec le point de vue des personnages. Si le discours "masculinisant" de Champlain cherche à implanter, à ensemencer, à faire couler le sang des martyrs pour qu'une Église germe et sorte de la terre, la narratrice, quant à elle, souligne la nature éphémère des projets réalisés en sol canadien. Dès les premiers chapitres sur Port-Royal-des-Champs, elle met l'accent sur le passage du temps, sur la célèbre abbaye qui, au moment de la rédaction, n'existe plus, ayant été absorbée par la terre: "[...] l'église allait s'ensevelissant par le travail du temps." $(O E, 21)$ Champlain rêve pareillement d'un corps à corps avec la terre: "[c]'est à Québec... c'est sous la terre canadienne que je désire dormir mon dernier sommeil." $(O E$, 59) Même quand il s'agit du glorieux travail de ces soldats du Christ, la fameuse résidence des Jésuites, Sainte-Marie-des-Hurons, le chaud foyer de la civilisation et de la foi, le texte nous rappelle

[qu'a]ujourd'hui depuis longtemps, la forêt a repris possession de cet endroit célèbre. [...] Ni la rivière ni les villages voisins, ne portent l'auguste nom de l'un de ces hommes apostoliques dont l'imagination aime à évoquer les ombres sur ce sol qu'ils ont illustré à jamais. $(O E, 184)$

Et encore, dans la description des bourgades huronnes de Sainte-Marie: "Quelques débris de palissades, des poteaux à demi consumés, de vastes clairières couvertes de cendres en indiquaient seulement la place." $(O E, 194)$ La symbolique du feu qui consume tout, qui ne laisse pas de traces, occupe une place grandissante dans l'évocation de l'abandon et de la mise à feu du fort Sainte-Marie, réduit à l'état d'" un vaste brasier dont la flamme s'élevait jusqu'au ciel" ( $O E, 200)$.

Force est de constater que dans $A$ l'oeuvre et à l'épreuve, le discours sur la fondation de la colonie est voilé, même équivoque, par moments. D'emblée une aventure masculine, la colonisation doit se faire en implantant la sève virile dans le sol canadien. L'héroïne du texte, Gisèle Méliand, après avoir aidé Charles Garnier dans sa vocation de missionnaire, soigne ses parents et, à leur mort, devient carmélite. À part quelques rares passages de la dernière partie, où elle reçoit des nouvelles de son bienaimé, elle se retire du récit. Il est vrai, comme le souligne Fernand Roy, que Garnier est déclaré martyr "grâce à la force de caractère de Gisèle", qui assure par ses prières que la grâce ne lui manque pas au moment du sacrifice final. De là à prétendre qu'elle est, "littérairement parlant, l'héroïne de papier du récit ${ }^{24}$, il y a plus qu'un pas: comment ne pas remarquer, en effet, l'absence d'une véritable présence féminine, du moins en terre canadienne, dans ce premier roman historique? Cette même absence voue la colonie à l'échec, car il n'y aura pas d'"ensemencement"

24. Fernand Roy, loc. cit., p. 195-196. 
sans la femme réelle. Ce difficile enracinement est préfiguré dans le texte par la force souterraine qui mine de l'intérieur le discours virilisant et refuse le colonisateur, maintenant tout au long du roman une tension entre la parole autoritaire et le discours des personnages, entre "ensemencer " et "effacer".

\section{Figures féminines, héroïne " dévirilisante »}

Le deuxième roman historique, L'oublié, commence sous le signe de l'héroïsme le plus chrétien avec l'exergue de La chanson de Roland: "Il est vrai que nous sommes peu nombreux, mais pour preux et hardis nous le sommes." $(O, 227)$ Le récit qui suit est celui des fondateurs et des pionniers de Ville-Marie (Paul de Chomedey, sieur de Maisonneuve, Marguerite Bourgeois, Jeanne Mance, Lambert Closse) qui n'ont pas lâché prise, ou plutôt "lâch[é] terre" devant les assauts iroquois. Élisabeth Moyen, enlevée par les Iroquois à la suite du massacre de sa famille, est échangée contre un chef autochtone capturé par Lambert Closse, sergentmajor du fondateur. Après sa convalescence, éblouie par le courage des fidèles colons et amoureuse de celui qui l'a sauvée, elle décide de rester à Ville-Marie au service de Jeanne Mance. Lambert Closse, quant à lui, ne veut pas au début se laisser détourner par des responsabilités familiales de son objectif premier: mourir en défendant sa sainte ville. Celui qu'on appelle le sauveteur de Ville-Marie changera pourtant d'avis après qu'Élisabeth à son tour lui aura sauvé la vie en désarmant un Iroquois malade qui essaie de le poignarder. Ils se marieront et vivront quelque temps ensemble mais sans grand bonheur. Ne pouvant pas participer à la bataille de Long-Sault, Closse se verra privé de son rôle de guerrier. Il mourra cependant dans une autre échauffourée contre l'ennemi autochtone, laissant derrière lui sa femme et sa fille.

L'image de Ville-Marie que nous offre L'oublié est empreinte d'une forte consonance féminine. Comme le souligne Ginette Michaud, "VilleMarie est considérée dans de nombreux textes du $\mathrm{xIX}^{\mathrm{e}}$ siècle comme objet de culte, comme objet de piété éminemment féminin ${ }^{25}$ ". Chez Laure Conan, c'est justement la Vierge Marie "qui nous protège et nous donne la victoire " $(O, 229)$, comme s'exclame le secrétaire de Maisonneuve, $M$. de Brigeac. Fidèle au discours messianique de l'époque, qui voit en l'histoire de la fondation de Ville-Marie un signe divin de la survie des francophones en Amérique du Nord, Maisonneuve souligne l'intervention de cette puissance suprême :

L'île de Montréal appartient à la sainte Vierge [...] Marie est notre Reine... et je voudrais voir sa statue briller dans les airs, au sommet de la montagne [...]

25. Ginette Michaud, "De la "Primitive Ville" à la Place Ville-Marie: lectures de quelques récits de fondation de Montréal,, Pierre Nepveu et Gilles Marcotte (dir.), Montréal imaginaire: ville et littérature, Montréal, Fides, 1992, p. 76. 
Tout notre espoir est en Elle, la Toute-Puissante... la Fidèle... l'Incomparable... la Radieuse ${ }^{26} \ldots(O, 230)$

À cela s'ajoute l'apparition d'un élément nouveau dans la poétique historique de l'auteure, soit la présence en sol canadien de femmes réelles, courageuses et héroïques, telles que Jeanne Mance et Marguerite Bourgeois.

Au moment même où tous les colons crient "Vive Notre-Dame " pour remercier celle-ci d'avoir sauvé Élisabeth, entre au fort sœur Marguerite Bourgeois, véritable incarnation de la Vierge. Élisabeth sera ensuite accueillie par sœur Bourgeois et mise entre les mains du deuxième "ange" de Montréal: l'admirable Jeanne Mance que l'historien M. Olier, cité dans le roman, décrit comme "environnée de la lumière divine comme d'un soleil" $(O, 241)$. Laure Conan construit ici une espèce d'hagiographie au féminin à l'intérieur de laquelle les notions de courage et de force morale sont modifiées pour inclure les actions de ces femmes intrépides et infatigables. Or, le caractère angélique de ces deux femmes n'annule en rien leur existence physique. En fait, elles offrent un exemple de conduite sur lequel la jeune Élisabeth peut se modeler. Jeanne Mance instruit leur nouvelle compagne de la contribution des femmes à la survie de la colonie: "Soyez confiante, conseille-t-elle à Élisabeth, car la Vierge nous garde [...] si elle voulait abandonner ses colons de Ville-Marie, elle ne leur aurait pas envoyé Marguerite Bourgeois." $(O, 245)$ La présence de cette illustre religieuse demeure une grande consolation pour Jeanne Mance, qui n'avait qu'un chagrin au moment de partir pour la NouvelleFrance: "[...] la pensée qu'il n'y aurait pas d'autres femmes qu'[elle] à Montréal." $(O, 247)$ Mais grâce à deux ouvriers qui refusaient de partir sans leur femme et à Geneviève qui s'est jetée de force dans le vaisseau, l'infirmière ne se trouve pas en terre exclusivement masculine. En remplissant son rôle de femme, elle sait se faire respecter: "[t]ous ces héros sont un peu enfants avec moi, ils m'obéissent tous." $(O, 247)$ À travers un réseau de références subtiles, le texte parvient même à faire une comparaison entre les deux femmes courageuses et le fondateur de Ville-Marie. Marguerite Bourgeois est venue au Canada avec M. de Maisonneuve; tout comme lui, elle a "quelques traits de ressemblance avec les anges" $(O, 246)$. Jeanne Mance, quant à elle, "tâche de faire comme M. de Maisonneuve, qui ne craint que $\operatorname{Dieu}^{27}$ " $(O, 246)$.

Cette communauté féminine influence profondément Élisabeth Moyen. Dans une lettre à une autre grande héroïne de l'histoire de la

26. On pourrait multiplier ad infinitum les passages de ce genre dans L'oublié: "Cette pensée de la Vierge animait tout, éclairait tout, à Ville-Marie. L'hérö̈sme opiniâtre se fondait dans son culte. L'image de Marie était brodée sur le drapeau; elle brillait sur le mur de chaque maison, comme une étoile; et, grâce à elle, une sorte de paix planait au-dessus de toutes les angoisses. " $(O, 255)$

27. C'est moi qui souligne. 
Nouvelle-France, mère Marie de. l'Incarnation, la jeune femme exprime son admiration pour cette colonie d'apôtres et de héros "qui vivent comme les fidèles de la primitive Église, en attendant l'heure du martyre" $(O, 253)$. Quoique son choix de rester à Montréal au lieu de retourner à Québec soit plus explicite dans la pièce qui a été tirée du roman ${ }^{28}$, il n'est pas tout à fait juste de conclure, comme le fait Roger Le Moine, que la jeune héroïne "ne gouverne pas son destin" $(O, 224)$. Tout en croyant aux volontés de Dieu, au chemin sur lequel la Providence l'a jetée, Élisabeth n'est pas entièrement passive. Elle s'obstine à aimer son sauveur, le grand défenseur de Ville-Marie, lui qui "apparaissait tellement au-dessus d'elle que la pensée la plus lointaine d'en être aimée un jour ne pouvait lui venir" $(O, 225)$. Son héros, Lambert Closse, a en effet donné son cœur à la Vierge, mais son choix du célibat semble découler d'une crainte singulière d'être "domestiqué", d'un refus des bonheurs de la terre - qui rappelle celui de Charles Garnier - mais aussi d'un refus du féminin, de la femme réelle qui pourrait saper son énergie. Il décline l'offre faite par Maisonneuve de lui faire bâtir une maison: "[j]e veux passer sur terre, sans laisser de traces, dit-il. Quand je m'en irai, je veux disparaître tout entier... oublié de tous... excepté d'Elle." $(O, 261)$ L'action héroïque d'Élisabeth l'oblige pourtant à renoncer à sa promesse faite à la Vierge. C'est au centre de L'oublié que se trouve la scène où, saisie d'une force soudaine, la jeune fille lui sauve la vie, ce qui provoque un renversement des rôles; la faible main de la femme protège le brave guerrier intouchable: "Lui, le fort, l'intrépide, elle l'avait vu à ses pieds, tout frémissant d'inquiétude et de tendresse. " $(O, 274)$

Dans L'oublié, l'auteure retravaille la configuration symbolique présentée dans $\dot{A}$ l'œuvre et à l'épreuve - le sang, le feu, la sève, le sol, les cendres, la tension entre "S'implanter" et "effacer" — mais le tout sur un plan plus complexe. Le discours "masculinisant" de domination, de fondation patriotique, est adouci par les figures de Closse et de Maisonneuve qui "lui aussi veut s'effacer, disparaitre comme les architectes de ces vieilles cathédrales, dont la terre admire les œuvres et ignore les noms" $(O, 262)$. Élisabeth, elle, sait vivre et cherche à s'ancrer dans le sol. Malgré sa fragilité - "Je suis une pauvre petite épave [...] une branche brisée" - elle pourrait prendre "à l'instant racine et sentir [...] la sève courir dans toutes ses fibres" $(O, 255)$. Sève de femme, soumission de

28. Laure Conan adaptera l'intrigue de L'oublié à la scène dans une pièce intitulée $A u x$ jours de Maisonneuve (1925). Dans son analyse de la version dramatique d'Élisabeth Moyen, Marilyn Baszczynski met en relief son refus de la passivité: "Orpheline, elle a la liberté de décider de son propre avenir, et elle refuse de se soumettre à l'autorité d'une nouvelle figure paternelle. À son retour à Ville-Marie, Élisabeth prend sa situation en main car c'est elle qui décide de rester à Ville-Marie au lieu d'aller à Québec vivre avec les d'Ailleboust. "Voir "Laure Conan: un thêatre au féminin au XIX ${ }^{c}$ siècle ", Theatre Research in Canada/Recherche canadienne du thêâtre, vol. XIV, n 1, 1993, p. 26. 
l'homme (face à la Vierge), tout semble s'inverser. Le sang et la sueur versés sur les champs se transforment en cendres. Élisabeth, blessée dans le combat avec l'Iroquois, prend la place du héros; elle verse son sang à la vue duquel Closse faiblit: "[1]e sang d'Élisabeth avait rougi ses mains: et ce sang versé pour lui, lui mettait de la neige et du feu dans les veines." $(O, 275)$ En fait, comme le précisera Ginette Michaud dans son étude des récits de fondation de Montréal, "[s]ang, neige, feu: on le pressent, ce ne sera pas surtout par la cohérence des métaphores que Ville-Marie se signalera, mais comme le lieu de rencontre des couples les plus contradictoires, les plus déchirés ${ }^{29}$ ", comme une vaste représentation antinomique:

Dans l'image de Ville-Marie viennent se mélanger, se tremper et se retremper, les vertus les plus hérö̈ques, les plus viriles, mais aussi, plus curieusement, les plus féminines: dès qu'on fait allusion à Ville-Marie, le sacrifice (de l'amour), l'abnégation et le don (de soi, à une Dame plus grande encore) sont constamment exaltés. Ville-Marie, et tout spécialement la figure de la Vierge, est le lieu où ces images opposées - eau et sang, neige et feu, blanc et rouge, virginité et sexualisation, féminité et héroïsme - se mêlent et échangent même leur caractère respectif, allant parfois même jusqu'à s'inverser: le héros viril féminisé par sa blessure, s'amollit en invoquant la Vierge, l'héroïne se virilise $[\ldots]^{30}$.

Non sans honte de rompre sa promesse à la Vierge, Closse, ayant surestimé ses forces, avoue son amour pour Élisabeth. Mais la double symbolique de forces opposées (de la neige et du feu) laissera pressentir sa déchirure interne. "Dévirilisée par sa femme après leur mariage, il abandonne la direction militaire du fort et se consacre à la terre afin d'y trouver la sève virile que la vie conjugale semble lui enlever: "[i]l voulait se mettre sérieusement à défricher: des entrailles de la terre, il voulait arracher pour son Élisabeth le pain, les fleurs, les fruit ${ }^{31}$." $(O, 283) \mathrm{La}$ frustration de Closse face à cette vie bucolique met en exergue l'incompatibilité entre la défense et la fondation de la colonie, entre ce qu'on pourrait appeler les visées masculines et féminines de l'aventure canadienne. À la différence d'Angéline de Montbrun et même de Gisèle Méliand, pour qui le bonheur est à chercher dans l'au-delà, Élisabeth Moyen compte jouir pleinement de la vie terrestre. Le fait qu'elle ait donné naissance à une fille est, dans ce sens, hautement significatif. À ce pouvoir maternel semble se heurter l'énergie belliqueuse qui sous-tend le personnage de Closse. Car se marier et fonder une famille constitue une trahison, une dérogation à son dévouement à la Vierge, cette essence féminine qui protège les colons et dont semble dépendre sa masculinité. Dans la poétique historique de Laure Conan, L'oublié marque ainsi une

29. Ginette Michaud, loc. cit., p. 61.

30. Ibid., p. 77-78.

31. C'est moi qui souligne. 
transition fondamentale pour le succès de la colonisation, à savoir celle entre la sublimation du divin féminin et la présence, à Ville-Marie, de femmes réelles qui survivent aux combattants masculins.

La complexité sémantique de ce roman ne se joue pas, comme dans À l'ouvre et à l'épreuve, dans le décalage entre les discours des personnages et celui de la parole autoritaire, mais à l'intérieur de celle-ci. Autrement dit, les interventions narratives du texte sont pleines d'ambiguité quant à l'importance tantôt édifiant, tantôt "démasculinisant " du féminin. C'est comme si, dans la pensée de l'auteure, le réseau de femmes hérö̈ques que formaient Marguerite Bourgeois, Jeanne Mance et Élisabeth Moyen dominait trop cette ville déjà connotée comme féminine. Les derniers chapitres du récit, centrés sur le martyre des dix-sept colons à LongSault, qui aura pour effet de sauver la colonie, rétablissent l'ordre traditionnel en remettant la gloire aux mains des pionniers masculins.

Ginette Michaud a bien montré l'importance du récit de la fondation de Ville-Marie pour le discours messianique de la fin du xix ${ }^{e}$ siècle. La survivance de moins d'une cinquantaine d'habitants malgré les implacables assauts autochtones préfigure la survivance du peuple francophone en Amérique du Nord. C'est l'histoire d'un miracle, de l'intervention divine.

[D]e la fin du XIX ${ }^{e}$ siècle jusqu'à la moitié du $x^{e}$, qui coïncide peu ou prou avec la célébration de son tricentenaire, c'est encore et surtout Ville-Marie qui sera chantée sur tous les tons, mais dans un registre décidément majeur: visions, délires, hyperboles, emphases, rien des excès de la rhétorique romantique ne sera épargné pour décrire la gloire de Ville-Marie, sa "floraison d'héroïsme", son "décor surnaturel ", son "atmosphère de légende "comme l'écrira son historien attitré au moment du tricentenaire, l'abbé Lionel Groulx ${ }^{32}$.

Le texte de Laure Conan souscrit pleinement à cette logique. Cependant, L'oublié tend à brouiller, à reconfigurer même, les rôles attribués aux femmes et aux hommes, de manière à redéfinir l'héroïsme masculin pour $\mathrm{y}$ inclure la force morale d'une Jeanne Mance et l'intervention physique d'une Élisabeth Moyen. La colonisation n'est plus seulement une entreprise masculine de domination et de combat contre l'ennemi, mais aussi une aventure féminine. Dans les portraits hagiographiques de Jeanne Mance et de Marguerite Bourgeois, Laure Conan nous dépeint des femmes qui accèdent à la sphère publique tout en conservant leurs grâces et leurs qualités altruistes d'infirmières et de directrices de conscience.

\section{Survivance, fidélité et tradition au féminin}

La sève immortelle commence par la bataille de Sainte-Foy, au lendemain de la Conquête. Jean Le Gardeur de Tilly y a été blessé. En

32. Ginette Michaud, loc. cit., p: 66. 
reprenant conscience, il apprend que "l'Anglais allait régner sur la terre où dormaient les héroïques pionniers français ${ }^{33}$ ". Le soldat achève sa convalescence, mais se désespère du sort de sa famille dépossédée de sa maison, ainsi que du sort de la Nouvelle-France récemment conquise. Distrait de sa peine par les soins de Thérèse d'Autrée, la fille d'un colonel français, il devient amoureux d'elle et s'apprête à quitter son pays pour la suivre en France, condition au mariage exigée par le colonel d'Autrée. Entre-temps, au manoir des Tilly, le frère de Jean sème grâce aux soins de sa cousine, Guillemette de Muy. Celle-ci a obtenu de Laycraft, un riche officier anglais, les graines nécessaires, mais refuse de l'épouser, espérant plutôt offrir son cœur à Jean. De Tilly, déchiré par le choix que lui impose le père de sa bien-aimée - il doit renoncer à sa patrie pour épouser Thérèse - , se laisse convaincre par sa mère de rester au Canada et d'épouser Guillemette. Thérèse, brisée, succombera aux suites d'une pneumonie dès son retour en France.

Dans les deux autres romans historiques, il s'agissait de fonder, de marquer le sol vierge canadien avec les traces du sang de ses martyrs. En ce sens, la vision qu'a Jean de Tilly de la Nouvelle-France se lit comme une confirmation de ces actes hérö̈ques. Dans ses rêves, Jean " voyait sortir de la terre, se lever suppliantes vers le ciel, les mains qui avaient défriché la forêt" $(S, 107)$. Il n'y a aucun doute pour lui que "les pas de Champlain avaient laissé sur la terre canadienne une empreinte que rien n'effacerait jamais" $(S, 204)$. Le souci de s'implanter, de s'enraciner, des premiers colons des textes précédents est remplacé par celui de la survivance de la race canadienne-française; l'espace restreint du fort est supplanté par le rêve d'un immense drapeau qui couvre la terre canadienne, le sol si longtemps français. La configuration symbolique (sang, feu, cendre, sol), lieu de forces oppositionnelles, se trouve simplifiée ici dans l'accent qui est mis sur la terre. L'homme canadien-français est comme une plante qui ne saurait se déplacer sans mourir; un rétrécissement des allégeances exclut ici la double appartenance à la France et au Canada. Le frère de Jean illustre bien le statut limitrophe du texte, à la frontière entre deux genres - roman historique et roman de la terre. Les exigences du roman du terroir, écrit sous le signe de la conservation, expliquent le dénouement du récit: Jean doit passer outre ses sentiments, faire le sacrifice de Thérèse, troquer son arme contre la houe et élever une famille en terre canadienne.

Quelles sont les conséquences de ce changement de cadre pour la contribution féminine à la préservation de la race? De façon générale, le discours national se caractérise de nouveau par une logique

33. Laure Conan, La sève immortelle, Euvres romanesques, tome III, Montréal, Fides, coll. "Nénuphar", 1975, p. 50. Désormais, les références à cet ouvrage seront indiquées par le sigle $S$, suivi du folio, et placées entre parenthèses dans le texte. 
"masculinisante". Jean de Tilly délire à propos des exploits hérö̈ques du chevalier de Lévis, personnage secondaire qui investit personnellement le jeune soldat de la mission de faire durer la race canadienne. Jean s'identifie même à Montcalm au moment de ses funérailles: "Je voyais descendre le cercueil dans la fosse... puis, je l'avais sur moi!... Ses clous me transperçaient." $(S, 125)$ De plus, le récit met en conflit le devoir patriotique et l'amour que Jean éprouve pour Thérèse:

Cette douceur, ce charme que la pensée de Mademoiselle d'Autrée avait
répandu sur sa tristesse, lui pèsent comme un remords: Il en ressentait une
honte et se jugeait petit, puéril. Avoir oublié la ruine de son pays, le malheur.
de tous les siens, pour songer à une jeune fille à peine entrevue, l'humiliait
profondément. $(S, 117)$

La présence de Thérèse lui fait oublier la honte de la Conquête, mais ses soins ont pour effet de l'affaiblir.

L'intrigue principale de La sève immortelle est compliquée toutefois par les autres personnages féminins du texte qui font preuve de courage et d'optimisme devant l'avenir incertain de la Nouvelle-France. À la différence de son fils, $\mathrm{M}^{\mathrm{me}}$ de Tilly n'est pas paralysée psychologiquement par l'état désespérant dans lequel se trouve la colonie conquise. Elle rappelle à son fils et à $M$. de Muy les devoirs de leur race: "Soyons catholiques et Français d'abord, et laissons le reste à Dieu." $(S, 167)$ Toutefois, c'est surtout Guillemette de Muy, la cousine de Jean, qui incarne cette fidélité aux origines. Elle refuse d'épouser le lieutenant Laycraft, riche soldat anglais à qui la famille de Tilly doit les outils nécessaires pour cultiver, ainsi que la permission de regagner leur maison. Comme le dit $\mathrm{M}^{\mathrm{me}}$ de Tilly, "ce n'est pas en mariant leurs filles à des protestants que les Canadiens conserveront la foi catholique dans le pays" $(S, 166)$. La scène du refus entre la femme canadienne-française fière et le soldat anglais bienveillant et compréhensif constitue bien entendu un des lieux communs de la littérature québécoise du XIx ${ }^{\mathrm{e}}$ siècle. Des Anciens Canadiens (1863) de Philippe Aubert de Gaspé père aux Exploits d'Tberville (1888) d'Edmond de Rousseau, en passant par Jacques et Marie (1864) de Napoléon Bourassa, on voit revenir le motif du choix ultime de l'héroïne qui sacrifie son amour à la fidélité nationale, séquence narrative qui renforce l'image traditionnelle de la femme comme gardienne des valeurs culturelles. La plus connue des scènes de refus est celle des Anciens Canadiens où Blanche d'Haberville est déconcertée par la 'demande en mariage d'Archibald Cameron de Locheill, ami d'enfance de la famille d'Haberville ${ }^{34}$.

34. Blanche s'écrie: "Vous m'offensez, capitaine Archibald Cameron de Locheill! ... Est-ce lorsque la torche incendiaire que vous et les vôtres avez promenée sur ma malheureuse patrie, est à peine éteinte, que vous me faites une telle proposition? Estce lorsque la fumée s'élève encore de nos masures en ruine que vous m'offrez la main 
Dans l'entretien entre Guillemette et Laycraft, il est impossible de ne pas voir une réécriture de la célèbre scène du refus, dans laquelle Laure Conan introduit une vision féminisée de l'histoire du Canada français. Les deux héroïnes justifient leur refus en évoquant les différences irréconciliables entre les Anglo-Saxons et les Canadiens français. Mais si Blanche personnifie l'innocence - "candide comme une paysanne qui n'a étudié ni ses sentiments ni ses réponses dans les livres, comme une campagnarde qui ignore les convenances d'une société qu'elle ne fréquente plus depuis longtemps $35_{n}$ - , Guillemette est la dépositaire de la mémoire des femmes de son pays. Face à l'impossibilité de la part de Laycraft de comprendre son attachement à un pays défait et destiné à mourir, elle lui répond: "Si vous connaissiez notre histoire [...] vous vous expliqueriez pourquoi les Canadiens ne veulent pas mourir." $(S, 172)$ Or, l'histoire qui compte pour elle ici, c'est celle des femmes, celle des Ursulines: "Savez-vous qu'au Canada les religieuses enseignantes ont précédé les défricheurs?", lui demande-t-elle en précisant que "comme un missionnaire, dont le nom m'échappe ${ }^{36}$, Mère de l'Incarnation aurait pu dater ses lettres: "du milieu d'un bois de huit cents lieues d'étendue"." $(S, 172)$ Le nom du missionnaire oublié et l'évocation de Mère de l'Incarnation dans les propos de Guillemette renversent la hiérarchie traditionnelle de l'historiographie, qui a l'habitude de retenir les exploits des hommes tandis que la contribution des femmes sombre dans l'oubli. L'exemple de mère Marie de l'Incarnation sert à la fois à redéfinir la nature du courage, afin de reconnaitre le rôle joué par ces femmes fortes du début de la colonie, et à fournir un modèle de comportement à celles qui devront prendre la relève en 1760 .

Guillemette puise également dans les souvenirs de sa propre famille d'une manière qui rappelle celle d'Angéline de Montbrun, en évoquant mère Sainte-Hélène (Charlotte de Muy) qui voyait dans la NouvelleFrance une ouvre d'héroïsme: "En voir la destruction lui fut une agonie. Elle mourut le lendemain de la bataille des Plaines" $(S, 173)$, comme si elle y avait combattu aux côtés de Jean de Tilly. En fait, le "cours d'histoire " que livre Guillemette à Laycraft n'est rien de moins que l'apogée de tout le projet d'histoire au féminin du Canada français amorcé dans les deux romans précédents. Commencé dans $\dot{A}$ l'œuvre et à l'épreuve sous la forme de l'exergue de Marie de l'Incarnation - "Le cœur humain est une forte pièce " $(O E, 17)$ - il sera poursuivi dans L'oublié où les figures de Marguerite Bourgeois et de Jeanne Mance soulignent l'importance de cette présence féminine pour la survie morale

d'un des incendiaires. "Voir Philippe Aubert de Gaspé, Les anciens Canadiens, Montréal, Bibliothèque Québécoise, 1988 [1863], p. 280.

35. Ibid., p. 281.

36. C'est moi qui souligne. 
et psychologique de la colonie. Guillemette de Muy, comme Élisabeth Moyen avant elle, ne se soustrait pas passivement à son rôle de gardienne de valeurs. En se dévouant au Canada, en nourrissant son amour pour la patrie, elle croit suivre les pas de plusieurs illustres devancières.

\section{Conclusion}

Selon la célèbre définition de l'abbé Casgrain, le texte digne du qualificatif national doit "moraliser le peuple en ouvrant son âme à tous les nobles sentiments, en murmurant à son oreille les noms chers à ses souvenirs, les actions qui les ont rendus dignes de vivre ${ }^{37}$ ". On aurait donc tort de prétendre que les trois romans historiques de Laure Conan ne remplissent pas les exigences du genre. Qu'il s'agisse d'une stratégie de légitimation (comme celle prônée par les premières journalistes) ou d'une quête d'autorité discursive (comme celle que revendique une lecture féministe), le passage du roman psychologique au roman historique n'a rien de dénaturé. En effet, l'auteure semble à l'aise dans le cadre du discours messianique qui traverse la pensée québécoise au tournant du siècle. La vision nationale qui se dégage de l'ensemble ne se caractérise pas, comme le soutient Maïr Verthuy, par une vision de plus en plus sombre de la destinée du Canada français, par une révolte contre l'autorité des pères et une patrie qui n'offre pas aux femmes la possibilité de la transformer ${ }^{38}$. Le recours à l'Histoire, comme cadre fictif et comme présence dans la mémoire des personnages féminins, autorise une autre interprétation des écrits de Conan. Alors qu'Angéline de Montbrun et $S i$ les Canadiennes le voulaient! indiquent une prise de distance par rapport à la patrie, liée en grande partie à un dégoût pour "l'arène souillée" de la politique, les romans historiques retournent au passé glorieux de la Nouvelle-France, où un panthéon bien établi de figures féminines divinisées - la Vierge, Marie de l'Incarnation, Marguerite Bourgeois et Jeanne Mance - autorise la libre expression de la fierté nationale.

Le patriotisme de Laure Conan ne se résume pas à une simple glorification des grandes figures féminines consacrées par l'histoire, lesquelles jouent en. fait des rôles secondaires dans la trame narrative de chaque roman, mais inclut la femme "moyenne", incarnée ici par Élisabeth Moyen, là par Guillemette de Muy. De la mère mythique, on passe ainsi à la vraie femme: l'épouse et la mère dont la présence en terre canadienne peut seule garantir la survie de la colonie. La critique subtile du discours viril sur la Conquête dans $\dot{A}$ l'œeuvre et à l'épreuve disparaît ainsi dans les deux romans suivants au fur et à mesure que sont reconfigurées les

37. Henri-Raymond Casgrain, op. cit., p. 369.

38. Maïr Verthuy, "Femmes et patrie dans l'œuvre romanesque de Laure Conan", Lori SaintMartin (dir.), L'autre lecture. La critique au féminin et les textes québécois, tome I, Montréal, XYZ éditeur, 1992, p. 39. 
notions de courage et d'héroïsme. Du point de vue de la narration, les moments extrareprésentationnels propres au genre historique, qui, chez Laure Conan, contestent le récit masculinisant de la colonisation, sont moins nécessaires lorsque l'auteure met en scène de véritables femmes. L'émergence de cette tradition historique au féminin ne cherche pas à supplanter l'Histoire des hommes mais à la compléter, à l'élargir, à la corriger. L'oscillation entre l'adhésion et l'opposition souligne une fois de plus la complexité de l'écriture des femmes, qui ne s'insère pleinement dans un paradigme établi et masculin, en l'occurrence l'épopée de la Nouvelle-France, qu'en le modifiant, même si ce n'est que pour mettre en relief l'héroïsme d'une hospitalière et la fidélité aux ancêtres féminins d'une jeune paysanne. 